\title{
Predicción del tamaño de prolactinomas mediante el valor plasmático de prolactina: la propuesta de una fórmula
}

\author{
Javier Calvo-Marín ${ }^{1 *}$, Juan Salazar-Borbón ${ }^{2}$, Heylin Montiel-Castillo ${ }^{3}$
}

\footnotetext{
${ }^{1}$ Médico Residente, Servicio de Endocrinología, Hospital México, Costa Rica

${ }^{2}$ Médico Interno, Servicio de Endocrinología, Hospital México, Costa Rica

${ }^{3}$ Médico Asistente Especialista, Servicio de Endocrinología, Hospital México, Costa Rica
}

Contacto con el autor: Dr. Javier Calvo-Marín, javiercalvomarin@ yahoo.com, (506) 8885-6423, Aptdo. 11401 Moravia, San José

Conflictos de interés: Los autores declaran no tener ningún conflicto de interés. La investigación se realizó en el Hospital México de la Caja Costarricense del Seguro Social con fondos de los propios investigadores, no hubo patrocinio externo alguno.

Fecha de recepción: 31/10/2015

Fecha de aceptación: 8/02/2016

\section{Resumen}

Objetivo: Describir la relación existente entre el tamaño tumoral y el nivel de prolactina en pacientes con diagnóstico de prolactinoma manejados en el servicio de endocrinología en un centro de atención especializada de Costa Rica.

Diseño: Estudio retrospectivo, observacional y descriptivo con los datos de un único centro.

Marco de referencia: En la evaluación del prolactinoma existe consenso en la correlación entre el nivel de prolactina y el tamaño del adenoma, no obstante, son escasos los reportes sobre la magnitud de esta relación y la posibilidad de establecer una ecuación que genere una aproximación del diámetro tumoral.

Participantes: Se incluyeron los pacientes con diagnóstico de prolactinoma manejados en el servicio de endocrinología de un centro hospitalario costarricense entre los años 2008 y 2014, en quienes se contaba con una medición de prolactina y un estudio por imágenes que lograra determinar la presencia de un adenoma hipofisario.

Intervenciones y mediciones: Para la determinación de prolactina se recurrió al inmunoensayo por electroquimioluminiscencia, y el tamaño tumoral del prolactinoma se definió en estudio por resonancia magnética o tomografía axial computarizada, con un lapso de separación no mayor a seis meses entre ellos.

Resultados: Se reclutaron 32 casos de pacientes con prolactinomas. La edad promedio fue de 32,8 años, con un $75 \%$ de mujeres y un $56,2 \%$ de macroprolactinomas; el tamaño tumoral promedio fue de 15,6 $\pm 12,3$ $\mathrm{mm}$ y la mediana de prolactina de $250,5 \mathrm{ng} / \mathrm{mL}$. Se obtuvo un coeficiente de correlación de Pearson de 0,822 $(\mathrm{p}<0,001)$, el cual dio base para la creación de dos fórmulas para la predicción del diámetro mayor de la lesión tumoral corregidas por la edad, la primera para prolactinemias menores de $500 \mathrm{ng} / \mathrm{mL}$ y otra para valores iguales o mayores a esta cifra.

Conclusiones: El presente estudio permitió desarrollar dos ecuaciones mediante las cuales se puede predecir el diámetro mayor tumoral aproximado a partir del valor inicial hormonal.

Palabras clave: prolactina, hiperprolactinemia, prolactinoma, adenoma hipofisario, infertilidad, galactorrea.

\section{Abstract}

Introduction: In the clinical evaluation of patients with prolactinomas, there is a consensus on the literature regarding the correlation between prolactin levels and tumor size; however, there are few reports that detail about the magnitude of this relationship and the possibility of establishing an equation that may estimate the adenoma diameter.

Material and methods: We conducted a retrospective, observational and descriptive study with data from a single center, which intended to verify the relationship between tumor size and prolactinemia. All of the included patients had measurements for prolactin blood levels and pituitary imaging with at least one diameter measurement of the adenoma.

Measurements and interventions: prolactin blood levels were determined by electrochemicaluminiscense immunoassay, and the size of the adenoma was defined 
by magnetic resonance imaging or computerized axial tomography scan, each study taken within a maximum of 6 months.

Results: Thirty-two cases of patients with prolactinomas were assessed. The average age of the patients was of 32,8 $\pm 13,1$ years, $75 \%$ were women and $56,2 \%$ were diagnosed with a macroprolactinoma. Mean tumor size was of 15,6 $\pm 12,3 \mathrm{~mm}$ and the median of prolactin level was $250,5 \mathrm{ng} / \mathrm{mL}$. We report a Pearson's correlation coefficient of 0,82 ( $p<0,001)$, showing a strong positive linear association between the prolactinemia and the tumor size. Through multiple linear regression analysis, we obtained two equations that allowed the prediction of the adenoma diameter given the prolactin level, adjusted by age.

Conclusion: We developed two equations that allowed us to estimate the largest tumoral diameter given the prolactin blood levels.

Keywords: prolactin, hyperprolactinemia, prolactinoma, pituitary adenoma, infertility, galactorrea.

\section{Introducción}

Los prolactinomas constituyen el tumor hipofisario secretor más común ${ }^{(1)}$ y suelen ser clasificados según su tamaño en microadenomas, si cuentan con un diámetro menor de $10 \mathrm{~mm}$ y macroadenomas si su tamaño es mayor a esta longitud. Los niveles séricos de prolactina en pacientes con este tipo de adenoma hipofisario usualmente guardan relación proporcional con el tamaño de la masa tumoral. De esta manera se pueden observar niveles mayores a $250 \mathrm{ng} / \mathrm{mL}$ y que pueden alcanzar los $10.000 \mathrm{ng} / \mathrm{mL}$ en pacientes con macroprolactinomas, hasta casos de $40.000 \mathrm{ng} / \mathrm{mL}$ en tumores gigantes ${ }^{(2,3)}$.

Resulta trascendente reconocer que existen otras causas de hiperprolactinemia, y en su proceso diagnóstico se deben tomar en cuenta ${ }^{(3,4)}$ causas fisiológicas y farmacológicas, enfermedades crónicas como insuficiencia renal, falla hepática o hipotiroidismo primario; además de compromiso de la vía inhibitoria tónica de la secreción de prolactina por lesiones que afectan la dinámica hipofisaria y estimulación del tejido mamario y pared torácica.

Con respecto a la relación entre niveles de prolactina y tamaño tumoral, se describen en los microprolactinomas valores que suelen rondar entre 50 y $300 \mathrm{ng} / \mathrm{mL}$, mientras que en macroprolactinomas, estos valores superan los $200 \mathrm{ng} / \mathrm{mL}$ y pueden alcanzar niveles de 5000 ng/mL o más ${ }^{(5)}$. No obstante, son pocos los reportes disponibles donde se define la magnitud exacta de esta relación, así como la posibilidad de contar con una fórmula matemática que permita predecir un estimado del tamaño esperable en el estudio por imágenes cuando se cuenta con el valor de prolactina.
A pesar de ser reconocida la relación existente entre los niveles de prolactina y el tamaño del prolactinoma, no se describe en la literatura una ecuación que estime un diámetro tumoral aproximado, tomando como base la prolactinemia y su interacción con otras variables como edad.

\section{Materiales y métodos}

\section{Diseño}

La investigación realizada es un estudio original, retrospectivo, observacional y descriptivo. Para la revisión de los expedientes clínicos se contó con la aprobación del Comité Local de Bioética, al cual se adscribe el centro médico de donde se obtuvieron los registros.

\section{Pacientes}

En el estudio fueron incluidos todos aquellos pacientes recibidos en el Hospital México en San José, Costa Rica, durante los años 2008 y hasta el 2013, con diagnóstico de hiperprolactinemia por prolactinoma, y que presentaran el resultado del nivel de prolactina con una separación no mayor a seis meses del estudio imagenológico, el cual podía consistir en una tomografía axial computarizada (TAC) o resonancia magnética nuclear (RMN) de silla turca con diagnóstico de adenoma hipofisiario y que incluyera al menos un diámetro de la lesión tumoral. Se excluyeron del análisis los casos de hiperprolactinemia para los cuales se identificó durante el seguimiento una causa secundaria que no fuera un adenoma hipofisiario funcionante productor de prolactina.

Se utilizó como fuente de información el expediente clínico de cada paciente, para quienes se recolectó la información correspondiente a sexo, edad, comorbilidades, medicamentos relacionados con hiperprolactinemia, síntomas de presentación; además de exámenes de laboratorio y gabinete como pruebas de función renal, perfil tiroideo, nivel de prolactina, estudio radiológico de silla turca (TAC, RMN). Por último, características de su manejo tales como uso de agonistas dopaminérgicos, cirugía y radioterapia.

\section{Métodos de laboratorio}

Para la determinación de prolactina se recurrió al uso de equipo Siemens Immulite $2000{ }^{\circledR}$ que establecía el nivel en ng/ $\mathrm{mL}$ mediante la técnica de inmunoensayo por electroquimioluminiscencia.

\section{Métodos estadísticos}

Para el análisis estadístico se utilizó el paquete de datos Small Stata 13,1. En el caso de las variables cualitativas se hizo uso de la prueba de Fisher para las relaciones entre sexo, sintomatología, comorbilidades, medicamentos y manejo; en sujetos divididos según tamaño tumoral en micro y macroadenomas. 
Tabla 1. Características clínicas en pacientes con prolactinomas

\begin{tabular}{|c|c|c|c|c|}
\hline & Total & Microadenoma & Macroadenoma & valor $p$ \\
\hline Cantidad & 32 & 14 & 18 & \\
\hline Edad (años) & $32,8 \pm 13,1$ & $27,1 \pm 6,7$ & $37,1 \pm 15,5$ & 0,035 \\
\hline Mujeres & $75 \%$ & $100 \%$ & $50 \%$ & 0,002 \\
\hline $\begin{array}{l}\text { Sintomatología } \\
\text { - Trastorno menstrual* } \\
\text { - Galactorrea* } \\
\text { - Cefalea } \\
\text { - Hipogonadismo } \\
\text { - Afección visual } \\
\text { - Infertilidad } \\
\text { - Libido disminuida }\end{array}$ & $\begin{array}{l}79,2 \% \\
70,8 \% \\
53,1 \% \\
28,1 \% \\
21,9 \% \\
12,5 \% \\
12,5 \% \\
\end{array}$ & $\begin{array}{c}78,6 \% \\
85,7 \% \\
57,1 \% \\
14,3 \% \\
21,4 \% \\
21,4 \% \\
0 \%\end{array}$ & $\begin{array}{c}88,9 \% \\
55,6 \% \\
50 \% \\
38,9 \% \\
22,2 \% \\
5,6 \% \\
22,2 \%\end{array}$ & $\begin{array}{l}0,075 \\
0,002 \\
0,722 \\
0,235 \\
1,000 \\
0,295 \\
0,113 \\
\end{array}$ \\
\hline Hipotiroidismo & $6,25 \%$ & $7,1 \%$ & $5,6 \%$ & 1,000 \\
\hline Uso de anticonceptivos & $6,25 \%$ & $14,3 \%$ & $0 \%$ & 0,183 \\
\hline Medicamentos & $6,25 \%$ & $7,1 \%$ & $5,6 \%$ & 0,438 \\
\hline
\end{tabular}

*Sólo documentado en mujeres

Según la distribución de los datos entre normal y no normal, se utilizó el $t$ test o la prueba de Mann-Whitney respectivamente, para la comparación de promedios entre variables de edad, tamaño de tumor y nivel de prolactina. Para cada una de estas pruebas, se consideraron significativos valores de p menores a 0,05.

En el caso de la construcción de la fórmula para la predicción del diámetro mayor de los adenomas productores de prolactina, se elaboró un modelo de regresión lineal múltiple ajustado por edad y el nivel de prolactina.

\section{Resultados}

Inicialmente se seleccionaron 48 pacientes en los cuales existía un diagnóstico de hiperprolactinemia por prolactinoma; no obstante 16 no contaban con una medición del diámetro del adenoma hipofisario en el estudio por imágenes realizado. Por tanto, se consiguió recabar los datos de 32 pacientes (tabla 1), con una edad promedio de 32,8 años y en su mayoría mujeres, las cuales representaron un $75 \%$ de la población. A la hora de efectuar la división según el tamaño de la lesión en hipófisis, se pudo notar que los pacientes con microadenoma tenían en el momento de la presentación una menor edad, y fueron en su totalidad mujeres; mientras que la distribución de sexo en pacientes con macroadenoma fue de 1:1 con una mayor edad cuando iniciaron el control $(37,1$ contra 27,1 años, $p=0,035)$.

En cuanto a la sintomatología, las molestias más frecuentes en el momento de presentación fueron galactorrea y trastorno en el ciclo menstrual (amenorrea u oligomenorrea) en las mujeres, mientras que en el hombre las manifestaciones más comunes incluyeron cefalea, hipogonadismo y alteraciones visuales. A la hora de comparar los hallazgos de los pacientes separados según el tamaño tumoral en micro y macroadenoma, únicamente se documentó diferencia estadísticamente significativa en la frecuencia de la galactorrea, siendo más prevalente en el microadenoma. Dentro de las causas de elevación hormonal, no asociadas a prolactinomas, se encontró una frecuencia de $6,25 \%$ tanto en hipotiroidismo, como en uso de anticonceptivos orales y otros fármacos que pueden elevar la prolactina.

Tabla 2. Tamaño tumoral y promedio de prolactina en pacientes con prolactinomas

\begin{tabular}{|c|c|c|c|c|}
\hline & Total & Microadenoma & Macroadenoma & valor $\mathbf{p}$ \\
\hline Cantidad & 32 & 14 & 18 & \\
\hline $\begin{array}{l}\text { Tamaño tumoral } \\
\text { (diámetro mayor en mm) }\end{array}$ & $15,6 \pm 12,3$ & $5,6 \pm 1,9$ & $23,3 \pm 11,5$ & $<0,001 *$ \\
\hline Promedio prolactinemia $(\mathrm{ng} / \mathrm{mL})$ & $1210 \pm 2420$ & $212 \pm 331$ & $1988 \pm 3023$ & $<0,001^{*}$ \\
\hline Mediana prolactinemia $(\mathrm{ng} / \mathrm{mL})$ & 250 & 119 & 633 & \\
\hline
\end{tabular}

*Ante una distribución no normal - se utilizó la prueba no paramétrica de Mann-Whitney 
Se documentó un promedio de tamaño tumoral de 5,6 $\mathrm{mm}$ contra $23,3 \mathrm{~mm}$ entre los micro y macroprolactinomas ( $\mathrm{p}<0,001)$, respectivamente (tabla 2), lo anterior acompañado de un promedio en niveles de prolactina de $212 \mathrm{ng} / \mathrm{mL}$ contra $1988 \mathrm{ng} / \mathrm{mL}(\mathrm{p}=<0,001)$. Se obtuvo un coeficiente de correlación de Pearson de 0,822 ( $\mathrm{p}<0,001)$ entre los valores de tamaño del tumor y el nivel de prolactina en sangre (figura 1).

Se elaboró un modelo de regresión lineal para establecer la fórmula aritmética que predijera el valor del diámetro mayor de la lesión tumoral según el nivel de prolactina, ajustado por la edad del paciente. Para describir mejor el comportamiento de ambas variables, se dividió en dos la población según el nivel de prolactinemia: si el valor era menor a $500 \mathrm{ng} / \mathrm{ml}$ (figura 2) y si el mismo era mayor o igual a $500 \mathrm{ng} / \mathrm{mL}$ (figura 3).

Figura 1. Correlación entre el tamaño tumoral y el nivel de prolactina

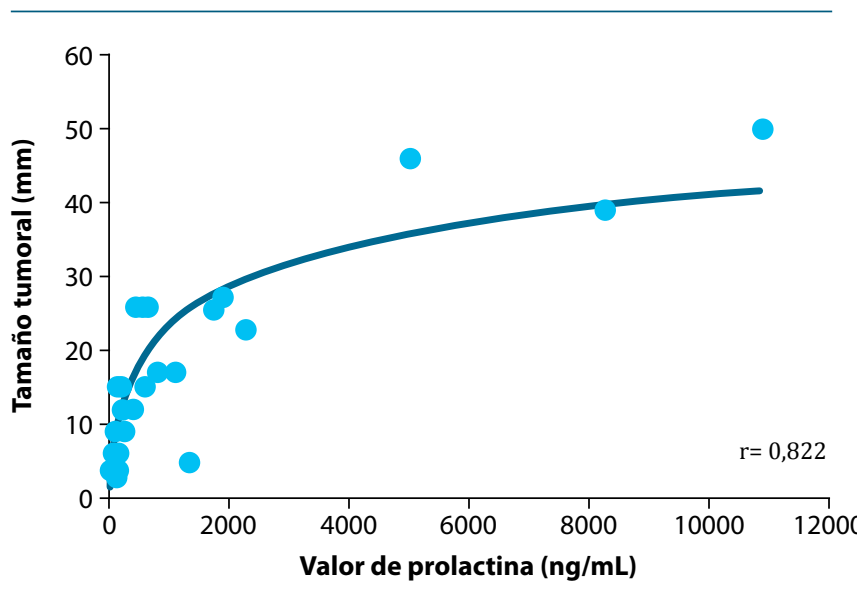

Figura 2. Cálculo de tamaño tumoral en pacientes con prolactinemia menor a $500 \mathrm{ng} / \mathrm{mL}$

\begin{tabular}{|c|c|c|c|c|}
\hline $\begin{array}{l}\text { DIÁMETRO } \\
\text { MAYOR }\end{array}$ & $\begin{array}{c}\text { prolactinemia } x \\
0,0290\end{array}$ & + & $\begin{array}{l}\text { edad } x \\
0,1264\end{array}$ & $-0,06$ \\
\hline
\end{tabular}

Figura3. Cálculo de tamaño tumoral en pacientes con prolactinemia igual o mayor a $500 \mathrm{ng} / \mathrm{mL}$

$\begin{aligned} & \text { DIAMETRO } \\ & \text { MAYOR }\end{aligned}=13.872+\begin{gathered}\text { prolactinemia } x \\ 0,00294\end{gathered}+\begin{gathered}\text { edad } x \\ 0,027\end{gathered}$

Adicionalmente, se recolectó el manejo que se decidió tomar en los pacientes con el diagnóstico de prolactinoma (tabla 3). En el 96,9\% se suministró un agente agonista dopaminérgico, en este caso la bromocriptina. Sin embargo por intolerancia a los efectos adversos o persistencia de la hiperprolactinemia, se recurrió a la cabergolina en el 59,4\% de los casos, con diferencia según el tamaño tumoral: $50 \%$ en microadenomas y $66,7 \%$ en macroadenomas ( $p=0,037$ ). Ningún microprolactinoma fue intervenido quirúrgicamente, y se recurrió a cirugía en el $16,7 \%$ de los casos de macroadenoma. Por último, no se documentó en este grupo de pacientes tratamiento mediante radioterapia.

\section{Discusión}

Se observó una correlación entre los valores de prolactina y el tamaño tumoral, lo cual permitió desarrollar dos modelos que predicen el tamaño aproximado del tumor a partir de los valores de prolactina en sangre.

En cuanto al tamaño tumoral, es reconocido el predominio de lesiones de mayor dimensión en la población masculina, y por tanto una menor frecuencia de microadenomas, tal como se encontró en este grupo de sujetos. Esto se debe a la presentación clínica de los prolactinomas en hombres, en los cuales es usual encontrar síntomas iniciales secundarios a la compresión generada por un macroprolactinoma ${ }^{(6,7)}$. La explicación para una presentación más temprana en mujeres tiene que ver con los trastornos menstruales, la infertilidad o galactorrea; molestias que las lleva a consultar de una forma más anticipa-

da, y por esta razón representan la mayoría de los pacientes con microadenomas ${ }^{(7,8)}$.

Con respecto al principal objetivo del estudio, se evaluó tanto el nivel de prolactina como el tamaño tumoral con la meta de reconocer la relación que existe entre estas dos variables y su utilidad para la práctica diaria. Como es posible apreciar en la tabla 2, existe una diferencia significativa en el tamaño tumoral y el nivel de prolactina según la división clásica de micro y macroadenomas hipofisarios. Ahora bien, al ahondar en esta relación y la posibilidad de analizar matemáticamente esta correspondencia entre los valores señalados previamente, se obtuvo un coeficiente de Pearson de 0,822 ( $\mathrm{p}<0,001$ ), el cual al acercarse a 1 refleja una relación positiva entre ambas variables (figura 1). Por tanto, se puede afirmar que a mayor

Tabla 3. Manejo realizado luego de diagnóstico de prolactinoma

\begin{tabular}{l|c|c|c|c}
\hline & Total & Microadenoma & Macroadenoma & valor p \\
\hline Cantidad & 32 & 14 & 18 & 0,438 \\
\hline Bromocriptina & $96,9 \%$ & $92,9 \%$ & $100 \%$ & 0,037 \\
\hline Cabergolina & $59,4 \%$ & $50 \%$ & $66,7 \%$ & 0,238 \\
\hline Cirugía & $9,4 \%$ & $0 \%$ & $16,7 \%$ & \\
\hline
\end{tabular}


nivel de prolactina, se espera un tamaño mayor en el estudio por imágenes utilizado para medir el prolactinoma. Dentro de los reportes encontrados en otras poblaciones, cuando se llegó a analizar la correlación mediante este mismo método se obtuvieron coeficientes similares: $0,805^{(9)}$ y $0,860^{(10)}$.

Una vez comprobada la correlación entre los niveles de prolactina y el tamaño tumoral, se establecieron las fórmulas detalladas en las figuras 2 y 3 , las cuales permiten estimar el diámetro mayor de un prolactinoma si se cuenta con el valor plasmático de prolactina y la edad del sujeto. Para tal fin se crearon dos modelos, tomando como corte el valor de prolactina de $500 \mathrm{ng} / \mathrm{mL}$, esta división se debe al comportamiento que tiene la relación conforme aumenta la prolactinemia, tal como se puede apreciar de forma gráfica en la figura 4. Utilizando esta herramienta, si se tuviera en una paciente de 25 años un valor de prolactina de $235 \mathrm{mg} / \mathrm{dL}$ se esperaría un adenoma hipofisario de $10 \mathrm{~mm}$; mientras tanto, en esa misma mujer, pero con una prolactina de $490 \mathrm{ng} / \mathrm{mL}$, se estimaría una medición aproximada de $16 \mathrm{~mm}$ para el diámetro mayor tumoral en el TAC o RMN.

Una de las realidades clínicas representada por esta ecuación matemática es el cambio en la magnitud del incremento del tamaño tumoral conforme los niveles de pro-

Figura 4. Relación entre los niveles de prolactina y el tamaño del prolactinoma dividida según prolactinemia
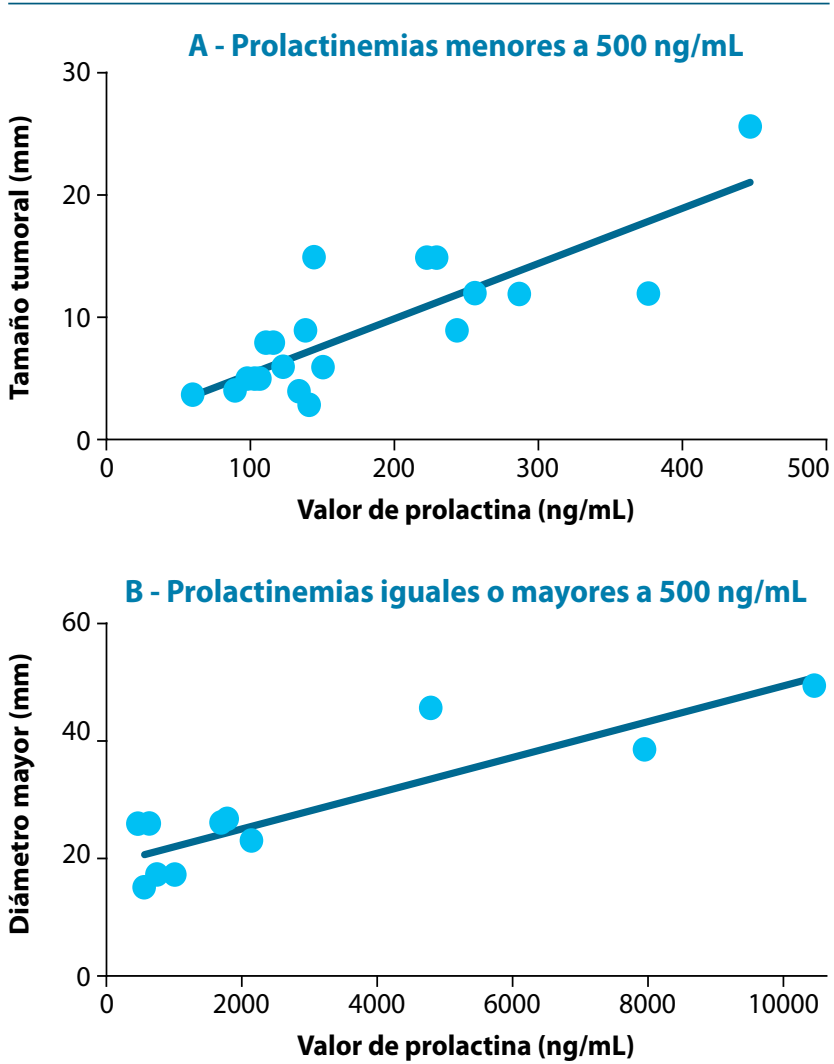

lactina superan un umbral determinado ${ }^{(11)}$, en este caso de $500 \mathrm{ng} / \mathrm{ml}$. Es decir, mediante las fórmulas se puede denotar cómo la diferencia en la prolactinemia de $300 \mathrm{ng} / \mathrm{ml}$ a 400 $\mathrm{ng} / \mathrm{mL}$ estima un incremento equivalente en el tamaño de la lesión (13,1 mm a 16 mm en el diámetro mayor, respectivamente), si se compara al efecto en el diámetro que implica una elevación de 3.000 a $4.000 \mathrm{ng} / \mathrm{mL}$ (23,6 mm a 26,6 mm, respectivamente).

A la hora de construir una ecuación cuyo fin es ser aplicada en la práctica clínica, existe posibilidad de medir su capacidad de acierto al correlacionar los resultados estimados luego del cálculo mediante la fórmula y el diámetro real que se obtiene en un estudio por imagen como TAC o RMN. De esta forma, se presenta la comparación de estos dos valores para los micro y macroadenomas en la población estudiada (figuras 5 y 6). El mismo ejercicio se podría realizar en un futuro, al aplicar la fórmula en sujetos portadores de prolactinomas no relacionados con los descritos en este grupo.

Con respecto a esta correlación obtenida, no se pueden olvidar dos posibles situaciones que cambian esta fuerte correlación entre el tamaño mayor del adenoma y el nivel de prolactina: el "efecto de gancho" y la macroprolactinemia. En el primer escenario existe una respuesta paradójica a la sa-

Figura 5. Comparación individual del diámetro tumoral mayor según la estimación mediante la fórmula y la longitud real reportada en el estudio por imágenes en pacientes con microprolactinomas
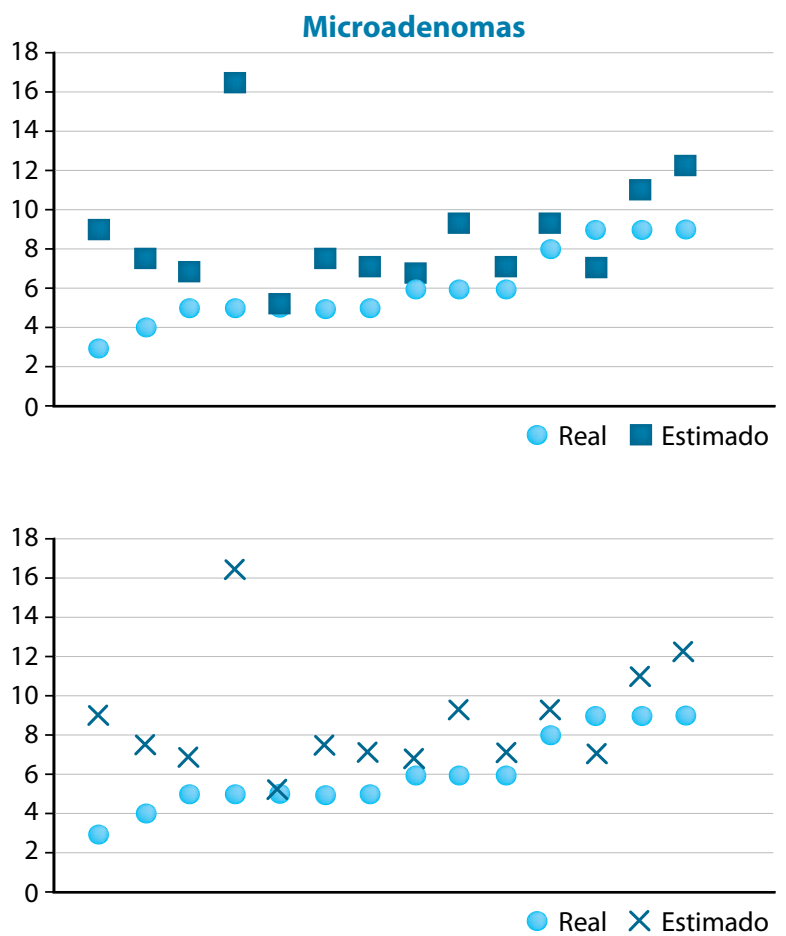
Figura 6. Comparación individual del diámetro tumoral mayor según la estimación mediante la fórmula y la longitud real reportada en el estudio por imágenes en pacientes con macroprolactinomas
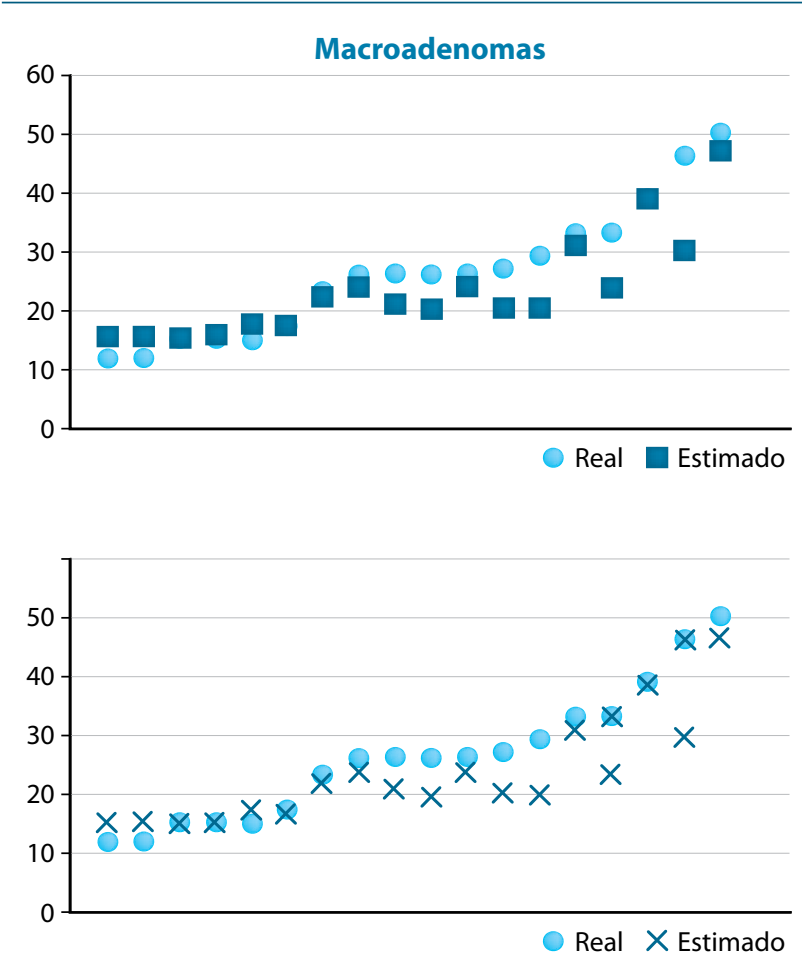

turación de la prueba de laboratorio debido a una excesiva cantidad de la prolactina que es medida como un antígeno por alguno de los ensayos que incluyen el uso de anticuerpos; para poder reconocer ese efecto se debe realizar una medición en diluciones cuando existe sospecha clínica de un mayor tamaño ${ }^{(12)}$. En el segundo reto diagnóstico puede darse la coexistencia de un incidentaloma hipofisario y una variante en el tamaño de la molécula de prolactina conocida como "big prolactina" o "big-big prolactina", en este caso se puede obtener un valor sérico de la hormona que no correlaciona con la lesión, debido a que el tumor no es el encargado de su secreción y por tanto no existe esa conexión entre los valores. Para resolver este escenario clínico, la historia clínica donde no existen marcados síntomas de hiperprolactinemia y una prueba de precipitación con polietilenglicol orientan para un acertado diagnóstico ${ }^{(13)}$.

Todas estas situaciones descritas previamente permiten convertir a la ecuación en una herramienta adicional para medir la importancia que tiene el nivel de prolactina, no sólo al inicio del proceso diagnóstico, sino también en los estudios de seguimiento con los cuales se vaya a controlar a estos pacientes. Además, un diámetro calculado por la fórmula que no concuerde con lo visualizado en los estudios por imágenes podría encender una alerta para descartar situaciones clínicas como la macroprolactinemia y el "efecto de gancho".

\section{Conclusión}

Se documenta con los datos presentados la existencia de una relación significativa entre el valor de la prolactina y el tamaño del prolactinoma, la cual permite generar una fórmula aritmética que brinda un estimado del diámetro mayor esperado en la lesión tumoral que más adelante debe ser reportada de forma definitiva en un estudio radiológico.

\section{Limitaciones}

Las mayores limitantes de este estudio derivan de su naturaleza retrospectiva. La principal fue la dificultad para contar con mediciones del tamaño del prolactinoma en el estudio por imágenes, situación que redujo en buena parte la población incluida para el análisis de datos. Esta dificultad puede llevar a un sesgo importante a la hora de evaluar el comportamiento de la lesión, sobre todo en los microadenomas, pues al ser menos frecuentemente reportada su dimensión en el estudio radiológico, disminuyó su inclusión en este reporte de datos. Llama la atención que en este el centro de salud del cual se obtuvieron los datos aún se tiene al TAC como el principal método diagnóstico, pues se llegó a recurrir a la RMN solamente en un $28,1 \%$ de los pacientes de la serie.

\section{Referencias}

1. Chen-Ku C. Guías para el diagnóstico y tratamiento de acromegalia, prolactinomas y enfermedad de Cushing. Acta Médica Costarricense 2004; 46(1) 25-36.

2. Iglesias $P$ et Diez J. Macroprolactinoma: a diagnostic and therapeutic update. QMJ 2013: 106(6) 495-504.

3. Klibanski A. Prolactinomas. New England Journal of Medicine 2010; 362: 1219-1222.

4. Rabinovich H. Clinical guidelines for diagnosis and treatment of prolactinoma and hyperprolactinemia. Endocrinología y Nutrición 2013; 60(6) 308-319.

5. Glezer A et Bronstein M. Prolactinomas. Endocrinology and Metabolism Clinics of North America 2015; 44(2): 71-78.

6. Iglesias P, Bernal C, Villabona C, Castro JC, Arrieta F, Díez J. Prolactinomas in men: a multicenter and retrospective analysis of treatment outcome. Clinical Endocrinology (Oxford) 2012; 77(2): 281-287.

7. Kars M. Update in prolactinomas. Netherlands: The Journal of Medicine 2010; 60(3): 104-112.

8. Colao A. The prolactinoma. Best Practice \& Research: Clinical Endocrinology \& Metabolism 2009; 23 (5): 575-596.

9. Cander, S. et al. Prolactin levels are correlated with tumor size and invasiveness in prolactinomas. Endocrine Abstracts 2012, 29: 1485.

10. Colao A, Sarno A, Cappabianca P, Briganti F, Pivonello R, Somma C, et al Gender differences in the prevalence, clinical features and response to cabergoline in hyperprolactinemia. European Journal of Endocrinology 2003; 148 (3): 325-331.

11. Molitch M. Pharmacologic Resistance in Prolactinoma Patients. Pituitary 2005; 8:43-52.

12. Frieze, T. Mong, D. Koops, M. "Hook effect" in prolactinomas: case report and review of literature. Endocr Pract. 2002; 8 (4): 296-303.

13. Glezer, A. Brasil, C. Delano, M. Vieira, J. Macroprolactina e Incidentaloma Hipofisário. Arq Bras Endocrinol Metab. 2001; 45 (2): 190-198. 